\title{
Dynamic contrast-enhanced MRI in breast cancer: A comparison between distributed and compartmental tracer kinetic models
}

\author{
Roberta Fusco $^{1,2}$, Mario Sansone ${ }^{1}$, Silvio Maffei $^{3}$, Nicola Raiano ${ }^{2}$, Antonella Petrillo ${ }^{2}$ \\ 1. Department of Biomedical, Electronics and Telecommunications Engineering, University "Federico II" of Naples, Italy. 2. \\ Department of Diagnostic Imaging, National Cancer Institute, Pascale Foundation, Naples, Italy. 3. Operative Unit of \\ Medical Physics, National Cancer Institute, Pascale Foundation, Naples, Italy.
}

Correspondence: Mario Sansone. Address: Department of Biomedical, Electronics and Telecommunications Engineering, University "Federico II" of Naples, via Claudio, 21, 80132, Naples, Italy. Telephone: 39-081-768-3807. Fax: 39-081768-3804. E-mail: msansone@unina.it.

Received: May 15, 2012

DOI : $10.5430 /$ jbgc.v2n2p23
Accepted: July 5, 2012

URL: http://dx.doi.org/10.5430/jbgc.v2n2p23

\section{Abstract}

Background/objectives: Dynamic Contrast Enhanced-Magnetic Resonance Imaging (DCE-MRI) is widely used in tumor diagnosis, staging and assessment of therapy response for different types of tumors, thanks to its capability to provide important functional information about tissue microvasculature. Tracer kinetic models used for estimating microcirculatory parameters can be broadly categorized as conventional compartmental (CC) or distributed- parameter (DP) models. While DP models seem to be more realistic, CC models (in particular the Tofts and the Brix models) have been widely used in clinical investigations over the past two decades. However, to date there is no direct comparison of CC vs DP models on real breast DCE-MRI data; moreover, a direct comparison between Tofts and Brix models, has not yet been reported on real breast data. Therefore, the purpose of this study was two-fold: on the one hand we analyzed the performance, on real breast DCE-MRI data, of CC vs DP models in terms of goodness-of-fit metrics; on the other hand we compared Tofts and Brix models on the basis of real breast DCE-MRI data.

Methods: Three models were compared: two CC models (the Tofts and the Brix models) and one DP model (the ATH model). We gathered data in two different scenarios: DCE-MRI with high temporal resolution obtained by means of a $k$-space under-sampling and data sharing method known as Time-resolved angiography With Stochastic Trajectories (TWIST) and DCE-MRI with low temporal resolution obtained by means of the Spoiled Gradient-Echo $k$-space scheme known as Fast Low Angle Shot (FLASH). The performances of the three models were evaluated by means of three goodness-of-fit metrics: the Residual Sum of Squares, the Bayesian Information Criterion and the Akaike Information Criterion on four breast DCE-MRI examinations.

Results: Although not conclusive, the results of this study suggest that the ATH model can achieve better fit in comparison to the Tofts and Brix models for TWIST data; and that the Brix model can achieve better fit with respect to the Tofts model for FLASH data.

Conclusion: Given the current typical settings of clinical breast DCE-MRI examinations, there seems not to be a clear advantage, in terms of goodness-of-fit, of ATH with respect to Tofts and Brix models; moreover, at lower temporal resolution the Brix model can achieve better fit than the Tofts model. 


\section{Key words}

DCE-MRI, Breast cancer, Tracer kinetics, Distributed parameters, Compartmental models

\section{I ntroduction}

Dynamic Contrast Enhanced-Magnetic Resonance Imaging (DCE-MRI) is widely used in tumor diagnosis, staging and assessment of therapy response for different types of tumors, thanks to its capability to provide important functional information about tissue microvasculature ${ }^{[1,2]}$. Evidence has shown that microcirculatory parameters (eg, perfusion, blood volume, mean transit time, and vessel permeability) derived from dynamic contrast-enhanced imaging may be linked to the aggressiveness or angiogenic potential of the tumor and may be useful for diagnosis and monitoring of cancer therapy outcome $^{[1-4]}$. These issues are particularly pertinent in breast carcinoma where there is a need to identify, before or early in treatment, those patients unlikely to respond to conventional therapy so that additional treatments may be given ${ }^{[5-10]}$.

For T1-weighted DCE-MRI, a variety of tracer kinetic models have been developed during the last two decades ${ }^{\text {[11-19] }}$. Conventional-Compartmental (CC) or Distributed-Parameter (DP) models ${ }^{[17-19]}$ are the two main categories of tracer kinetic models used for perfusion parameter estimation in DCE-MRI. Many investigations, especially clinical studies, have been published using CC models: in particular the Tofts ${ }^{[8-10,13,14,16]}$ and the Brix models ${ }^{[13,20-22]}$. The former, proposed by Tofts and Kermode ${ }^{[11]}$, used a population-based AIF with two exponentials, that was drawn from the literature concerning excretion of Gd-DTPA in the normal population (Weinmann et al. ${ }^{[25]}$ ). On the contrary, the model originally proposed by Brix et al. ${ }^{[15]}$ used a single exponential AIF by including one more parameter in the model. The Tofts and the Brix models (which have been shown to be both descending from a complete $\mathrm{CC}$ model ${ }^{[8]}$ ) have been widely used due to their simplicity ${ }^{[14,26]}$. However, these methods are only applicable when permeability-limited conditions are met ${ }^{[8]}$. Moreover, although some results in separating benign from malignant lesions have been obtained ${ }^{[26]}$, their acceptance in the clinical environment is not yet fully achieved, probably because of the not completely clear interpretation of some parameters (such as $\mathrm{K}^{\text {trans }}$ ) which does not allow to separately consider flow $(\mathrm{F})$ and permeability surface area product (PS).

On the other side, several authors ${ }^{[12,18,24,29]}$ have reported that DP models could allow a more complete analysis of kinetic parameters: Larson et al. ${ }^{[18]}$ stated that CC models do not possess sufficient realism, because tracer concentration gradients within compartments are assumed to be zero at all times and consequently the tracer is assumed to distribute instantaneously on arrival in each compartment. On the contrary, in DP models the concentration gradients in the vascular compartment are considered as a function of both time and space. The first DP model for two compartments was proposed by Johnson and Wilson ${ }^{[7]}$ under the name of tissue homogeneity (TH) model; later, St Lawrence and Lee ${ }^{[12]}$ proposed a simplified version with an adiabatic approximation of the tissue homogeneity model (ATH). The ATH model allows direct quantification of flow $(\mathrm{F})$, extraction fraction $(\mathrm{E})$, the clearance constant $\left(\mathrm{k}_{\mathrm{ep}}\right)$ and the mean capillary transit time $\left(\mathrm{T}_{\mathrm{c}}\right)$. Here, rather than defining the composite parameter $\mathrm{K}^{\text {trans }}$, it is possible to separate the flow from permeability.

To date, studies reporting comparison between CC and DP models in the DCE-MRI context have been few. Donaldson et al. ${ }^{[36]}$ provided a comparison among four different models: Tofts model, extended Tofts model with plasma fraction volume contribution, an uptake model and a general two-compartment exchange model (2CXM) in the carcinoma of cervix. Their results suggested that the assumption of negligible plasma mean transit time is not appropriate in this context and the 2CXM is better suited for its analysis than the Tofts models. This demonstrated the importance of selecting an appropriate tracer kinetic model in DCE-MRI. Li et al. ${ }^{[29]}$ compared four different models by applying four statistical measures (chi-square test, Durbin-Watson statistic, Akaike Information Criterion (AIC) and Bayesian Information Criterion (BIC)) to assess their capability to describe DCE-MRI data obtained in breast cancer. They examined the fast exchange limit model with (FXL_vp) and without (FXL) a plasma component, and the fast and slow exchange regime 
models (FXR and SXR, respectively). They reported that the FXL_vp and the FXR models provided the most complete statistical description of dynamic contrast-enhanced MRI time courses for the patients selected in their study.

To the best of our knowledge, apart from the simulation-based study by Zwick et al. ${ }^{[13]}$, there is no direct comparison between Tofts and Brix models on real data; moreover, there is no direct comparison of CC models and DP models on real breast DCE-MRI data.

With the previous considerations in mind, the objective of this study was two-fold: on the one hand we compared Tofts to Brix, on the other hand we aimed to compare DP (ATH) to CC models (Tofts and Brix); both comparisons were based on real breast DCE-MRI data.

It should be pointed out that in this study we considered only a few CC models that have been previously widely used in breast DCE-MRI (Tofts and Brix). Therefore, many other CC models have not been considered here (such as for example, the Patlak model ${ }^{[23]}$, which was developed originally for blood-brain-barrier exchange, or the general two-compartment exchange model as analyzed by Donaldson ${ }^{[36]}$ ).

We analyzed breast DCE-MRI data from 4 subjects with histologically proven invasive ductal carcinoma; they underwent DCE-MRI examinations in two different protocols: DCE-MRI with high temporal resolution obtained by means of a $k$-space under-sampling and data sharing method known as Time-resolved angiography With Stochastic Trajectories (TWIST) and DCE-MRI with low temporal resolution obtained by means of the common Spoiled Gradient-Echo $k$-space scheme (Spoiled Gradient Echo knew as Fast Low Angle SHot(FLASH) ${ }^{[30]}$. This was done because the ATH model requires a sampling interval lower than the mean capillary transit time $\left(\mathrm{T}_{\mathrm{c}}\right)$. FLASH data were used to perform the comparison between Brix and Tofts models in a typical clinical setting (as in previous studies). Models have been compared using different goodness-of-fit metrics (Residual Sum of Square (RSS), BIC, AIC). Moreover, a study of reliability of fit parameters estimation was performed.

\section{Materials and methods}

\subsection{Patients data and DCE-MRI acquisition}

Four women (38-53 age) with histologically proven invasive ductal carcinoma underwent imaging with a $1.5 \mathrm{~T}$ scanner (Magnetom Symphony, Siemens Medical System, Erlangen, Germany) equipped with dedicated 4-channel breast coil.

Dynamic images were obtained before and after intra-venous injection of $0.1 \mathrm{mmol} / \mathrm{kg}$ body weight of a Gd-DOTA (Dotarem, Guerbet, Roissy CdG Cedex, France; $r_{1}=4.5 \times 10^{-3} \mathrm{mM} / \mathrm{s}$ ). Automatic injection system was used (Spectris Solaris EP MR, MEDRAD, Inc., Indianola, Pennsylvania) and injection constant flow rate was set to $2 \mathrm{~mL} / \mathrm{s}$ followed by a flush of $10 \mathrm{~mL}$ saline solution at the same rate.

For two women DCE T1-weighted FLASH 3-D coronal images were acquired (TR/TE: 9.8/4.76 ms; flip angle: 25 degrees; matrix: $256 \times 128$; thickness: $2 \mathrm{~mm}$; gap: 0; acquisition time: $56 \mathrm{~s}$; 80 slices spanning entire breast volume, 11 temporal series). Three pre-contrast volumes were acquired with different flip angles (7, 12 and 25 degrees) in order to obtain T10 map in accordance with the standard methods proposed in literature ${ }^{[12]}$.

For the other two women DCE T1-weighted TWIST 3-D coronal images were acquired (TR/TE: 3.08/1.18 ms; flip angle: 25 degrees; matrix: $320 \times 290$; thickness: $2 \mathrm{~mm}$; gap: $0 \mathrm{~mm}$; acquisition time: $6.2 \mathrm{~s}$; 64 slices spanning entire breast volume; 88 temporal series; $\mathrm{pA}: 0.20$, pB: 0.20 ). The choice of $\mathrm{pA}$ and $\mathrm{pB}$ was based on the results of ${ }^{[30]}$. Five pre-contrast volumes were acquired with different flip angles $(7,12,15,20$ and 25 degrees) in order to obtain T10 map in accordance with the standard methods proposed in literature ${ }^{[33]}$. 


\subsection{Estimation of tracer concentration}

Using a spoiled gradient echo acquisition, the signal intensity at time $\mathrm{t}\left(\mathrm{S}_{\mathrm{t}}\right)$ from a tissue having longitudinal relaxation time $\mathrm{T} 1$ and transversal relaxation time $\mathrm{T} 2 *$ can be described by eq. $1^{[31]}$ :

$$
S_{t}=M_{0} \sin (\alpha) \frac{1-\exp \left(-T R / T_{1}(t)\right)}{1-\cos (\alpha) \exp \left(-T R / T_{1}(t)\right)} \exp \left(\frac{-T E}{T_{2}{ }^{*}}\right)
$$

where $\alpha$ is the flip angle; TE is the echo time; TR is the repetition time; $\mathrm{M}_{0}$ describes the scanner gain and proton density.

To perform quantitative DCE-MRI data analysis, the time varying longitudinal relaxation time, $T_{1}(t)$, must be related to the concentration of contrast agent $(C A)$ in the tissue, $\mathrm{C}_{\mathrm{t}}(\mathrm{t})$. Usually, a linear relationship between the two quantities is assumed ${ }^{[32]}$ (eq. 2):

$$
R_{1}(T)=\frac{1}{T_{1}(T)}=r_{1} C_{t}(t)+R_{10}
$$

where $R_{10}$ is the $R_{1}$ value of the tissue before CA administration and $r_{1}$ is the relaxivity of the CA. $R_{10}=1 / T_{10}$ may be estimated using several gradient echo images with different flip angles taken before contrast injection ${ }^{[33]}$. Rearranging eq. 1 yields:

$$
Y=X \exp \left(\frac{-T R}{T_{1}}\right)-M_{0}\left(1-\exp \left(\frac{-T R}{T_{1}}\right)\right) \exp \left(\frac{-T E}{T_{2}^{*}}\right)
$$

where $\mathrm{Y}=\mathrm{S}_{\mathrm{t}} \sin (\alpha)$ and $\mathrm{X}=\mathrm{S}_{\mathrm{t}} \tan (\alpha)$. Hence a plot of $\mathrm{Y}$ against $\mathrm{X}$ for a range of flip angles will result in a straight line, and $\mathrm{T}_{10}$ may be estimated from the slope.

\subsection{Tracer kinetic models}

Many tracer kinetic models have been developed for DCE-MRI data ranging from simplified two-compartment models to more physiologically plausible distributed parameter models ${ }^{[9-12,34]}$. In this section we introduce the models and the associated contrast agent input functions which we have considered in this study.

\subsubsection{Tofts model}

In the Tofts model ${ }^{[11]}$ the concentration of CA within plasma $\left(\mathrm{C}_{\mathrm{p}}(t)\right)$ after the injection of a bolus of Gd (also called Arterial Input Function, AIF) was assumed to be the one measured in normal control subjects by Weinmann ${ }^{[25]}$. This was fitted to a bi-exponential decay, which is expected from the compartmental theory:

$$
C_{p}(t)=D\left(a_{1} \exp \left(-m_{1} t\right)+a_{2} \exp \left(-m_{2} t\right)\right)
$$

where $\mathrm{D}$ is the dose $(\mathrm{mmol} / \mathrm{kg})$. The fitted values were $\mathrm{a}_{1}=3.99 \mathrm{~kg} / \mathrm{L}, \mathrm{a}_{2}=4.78 \mathrm{~kg} / \mathrm{L}, \mathrm{m}_{1}=0.144 \mathrm{~min}^{-1}, \mathrm{~m}_{2}=0.0111 \mathrm{~min}^{-1}$. The time course of tissue concentration was modeled as follows:

$$
C_{t}(t)=K_{\text {trans }} C_{p}(t) \otimes \exp \left(-k_{e p} \cdot t\right)+v_{p} C_{p}(t)
$$

Where $\mathrm{K}^{\text {trans }}\left[\mathrm{min}^{-1}\right]$ is the volume transfer constant from plasma to extracellular-extravascular space (EES); $\mathrm{k}_{\mathrm{ep}}\left[\mathrm{min}^{-1}\right]$ is the diffusion rate constant from EES to plasma, and $\mathrm{C}_{\mathrm{p}}(\mathrm{t})$ was obtained by eq. 4 . In particular $\mathrm{K}^{\text {trans }}$ is associated to both the vessel permeability and blood flow, $\mathrm{k}_{\mathrm{ep}}$ is linked to the duration of the wash-out phase, $\mathrm{v}_{\mathrm{p}}$ is plasma volume fraction ${ }^{[37]}$. $\mathrm{K}^{\text {trans }}$ and $\mathrm{k}_{\mathrm{ep}}$ satisfy eq. (6) where $\mathrm{v}_{\mathrm{e}} \in(0,1)$ is the volume fraction occupied by EES. 


$$
v_{e}=\frac{K_{\text {trans }}}{k_{e p}}
$$

\subsubsection{Brix model}

In the Brix et al. model ${ }^{[15]}$ the signal enhancement was assumed to be proportional to the concentration of CA in the tissue, the plasma concentration is fitted with single exponential decay characterized by a rate constant $\mathrm{k}_{\mathrm{el}}\left[\mathrm{min}^{-1}\right]$. During the infusion of contrast material the $\mathrm{CA}$ concentration $\mathrm{C}_{\mathrm{t}}$ is equal to:

$$
C\left(t, A, k_{e l}, k_{e p}\right)=\frac{A}{k_{e l}-k_{e p}}\left(\frac{1-\exp \left(-k_{e p} t\right)}{k_{e p}}-\frac{1-\exp \left(-k_{e l} t\right)}{k_{e l}}\right)
$$

where $\mathrm{A}\left[\mathrm{mmol} \mathrm{min}^{-1} \mathrm{~L}^{-1}\right]$ is the initial slope of the curve.

\subsubsection{ATH model}

According to the adiabatic approximation to the tissue homogeneity (ATH) model ${ }^{[12]}$, the concentration of CA in tissue, $\mathrm{C}_{\mathrm{t}}(\mathrm{t})$, is equal to the convolution of the arterial input function, $\mathrm{C}_{\mathrm{p}}(\mathrm{t})$, and the tissue impulse response function $\mathrm{H}(\mathrm{t})$ :

$$
\begin{gathered}
H(t)=F_{P}, t \leq \mathrm{T}_{\mathrm{c}} \\
H(t)=E \cdot F_{p} \exp \left(-\frac{E \cdot F_{p}}{v_{e}}\left(t-T_{c}\right)\right), t>\mathrm{T}_{\mathrm{c}}
\end{gathered}
$$

The ATH is more complex than the Tofts and Brix model. It accounts for different contributions from the plasma flow rate $F_{p}\left[\mathrm{~mL} 100 \mathrm{~g}^{-1} \mathrm{~min}^{-1}\right]$, extraction fraction through first-passage $\mathrm{E}$, mean capillary transit time $\mathrm{T}_{\mathrm{c}}$ [min], and interstitial volume fraction $\mathrm{v}_{\mathrm{e}}$.

The relationship with the above quantities $\mathrm{K}^{\text {trans }}$ and $\mathrm{v}_{\mathrm{p}}$ can be obtained with following equations:

$$
\begin{aligned}
& K^{\text {trans }}=E \cdot F \\
& v_{p}=T c \cdot F
\end{aligned}
$$

In this study we estimated $\mathrm{C}_{\mathrm{p}}(\mathrm{t})$ by means of non-linear fitting of the arterial flux measured directly on the images using the computationally efficient model recently proposed by ${ }^{[27]}$ :

$$
C_{p}(t)=A_{B} \cdot t \cdot \exp \left(-m_{B} \cdot t\right)+A_{G} \cdot\left(\exp \left(-m_{G} \cdot t\right)-\exp \left(-m_{B} \cdot t\right)\right)
$$

with $A_{B}=a_{B}-a_{B} a_{G} /\left(m_{b}-m_{G}\right)$ and $A_{G}=a_{B} a_{G} /\left(m_{b}-m_{G}\right)^{2}$ : in fact, the model proposed ${ }^{[27]}$ was based on decomposition of the AIF in a bolus term and a body transfer function term; $\mathrm{m}_{\mathrm{B}}, \mathrm{a}_{\mathrm{B}}$ and $\mathrm{m}_{\mathrm{G}}, \mathrm{a}_{\mathrm{G}}$ are the transfer rate and the amplitude of two terms respectively ${ }^{[27]}$.

\subsection{I mage and data analysis}

Manual segmentation was performed by an expert radiologist on the fat suppressed image obtained subtracting the basal pre-contrast image from one post-contrast image. The segmentation was performed by means of the OsiriX v.3.8.1 $3^{[46]}$.

Two scenarios were compared: the first with high temporal resolution (TWIST acquisition with $6.2 \mathrm{~s}$ sampling interval); the second with low temporal resolutions (FLASH acquisition with 56 s sampling interval). 
$\mathrm{T}_{10}$ maps were calculated using eq. (3). However, for all the voxels within a segmented ROI the $\mathrm{T}_{10}$ median value was used for CA quantification. This approach was inspired by Schabel et al. $2010^{[26]}$.

For $C_{p}(t)$ fitting using eq. (10) breast arteries have been manually selected. The starting estimates for $A_{B}, A_{G}, m_{B}, m_{G}$ were chosen on basis of results of ${ }^{[27]}$.

The starting estimates for non linear regression of the tracer kinetic models were chosen using the kinetic parameters values found in literature ${ }^{[15,20,38,39]}$ (see Table 1).

Table 1. Starting estimates for nonlinear regression

\begin{tabular}{lllllllll}
\hline & $\mathbf{K}^{\text {trans }}\left[\mathbf{m i n}^{-1}\right]$ & $\mathbf{k}_{\text {ep }}\left[\mathbf{m i n}^{-1}\right]$ & $\mathbf{v}_{\mathbf{p}}$ & $\mathbf{A}[\mathbf{m m o l} / \mathbf{k g}]$ & $\mathbf{k}_{\text {el }}\left[\mathbf{m i n}^{-1}\right]$ & $\mathbf{F}\left[\mathbf{m L} \mathbf{1 0 0} \mathbf{g}^{-1} \mathbf{m i n}^{-1}\right]$ & $\mathbf{E}$ & $\mathbf{T}_{\mathbf{c}}[\mathbf{s}]$ \\
\hline Tofts & 0.5 & 0.5 & 0.05 & & & & & \\
Brix & & 0.5 & & 0.001 & 0.144 & & 0.7 & 6 \\
ATH & 0.5 & & & & 21 & \\
\hline
\end{tabular}

For the parameters to fall in physiologically meaningful ranges, upper bounds were imposed on $\mathrm{K}^{\text {trans }}, \mathrm{v}_{\mathrm{p}}, \mathrm{v}_{\mathrm{e}}, \mathrm{A}, \mathrm{k}_{\mathrm{el}}, \mathrm{F}_{\mathrm{p}}, \mathrm{E}$,

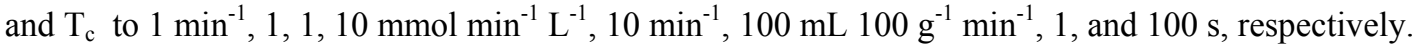

The data acquired with TWIST protocol were used to compare Tofts, Brix and ATH models while the data acquired with FLASH protocol were used to compare Tofts and Brix models.

Data fitting was performed using constrained nonlinear curve fitting in Matlab (v. 7.0; MathWorks, Natick, MA).

\subsubsection{Goodness of Fit}

A cost function commonly used for quantifying the goodness-of-fit between models and data is given by the residual sum of squares (RSS):

$$
R^{2}=\sum_{i=1}^{N}\left(y_{i}-C_{t}(i)\right)^{2}
$$

where $\mathrm{N}$ denotes the number of observations, $y_{i}$ is the observation at time $\mathrm{i}$ the $\mathrm{C}_{\mathrm{t}}(\mathrm{i})$ is the associated fitted value. The higher the $\mathrm{R}^{2}$ value the higher the discrepancy between the data and the model. However, this metric is not well suited to compare models with different number of parameters (as is the case for Brix vs ATH or Tofts vs ATH). To this aim we used the Bayesian Information Criterion ${ }^{[40]}$ and corrected Akaike Information Criterion (AICc) ${ }^{[41]}$.

Both AICc and BIC make a balance between the goodness-of-fit and the model complexity in a similar manner (they have a common statistical basis in the Kullback-Leibler divergence ${ }^{[41]}$ ); however, the BIC applies a heavier penalty on the model complexity:

$$
B I C=N \log \left(R^{2} / N\right)+p \log (N)
$$

The AIC is computed via eq. 13:

$$
A I C=N \log \left(R^{2} / N\right)+\frac{2 p(p+1)}{N-p-1}
$$




\subsubsection{Reliability of Fit Parameters}

In order to evaluate the reliability of fit parameters a Monte Carlo simulation was performed. Per each model and per each voxel, the estimated parameters were used to compute the corresponding tissue concentration curve by means of eq. 5-7-8. After, 100 repetitions of Gaussian noise were added to each curve (standard deviation calculated as square of RSS). Per each repetition a new parameter estimate was obtained. Using the 100 estimates we obtained the standard deviation per each parameter of a specific model, corresponding to a specific voxel. Subsequently, the median value of all the standard deviations for a specific parameter was calculated. This value should represent the confidence interval for the estimate of that specific parameter.

\section{Results}

Figure 1 shows an example of FLASH data: in particular the T1-w image, the fat-suppressed image obtained subtracting the basal signal from $5^{\text {th }}$ post contrast image and the ROI segmented by an expert radiologist. Similarly, figure 2 shows an example of TWIST data.

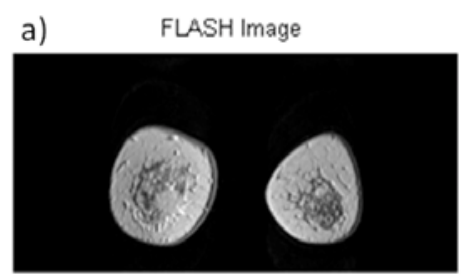

b) Fat-suppressed Image
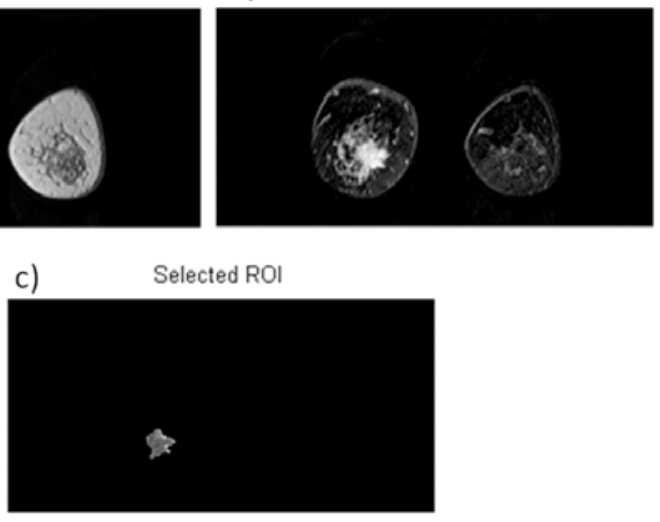

Figure 1. FLASH data: a) T1-w example image; b) Fat-suppressed image obtained subtracting the basal image from the 5th post contrast image; c) ROI selected by an expert radiologist.
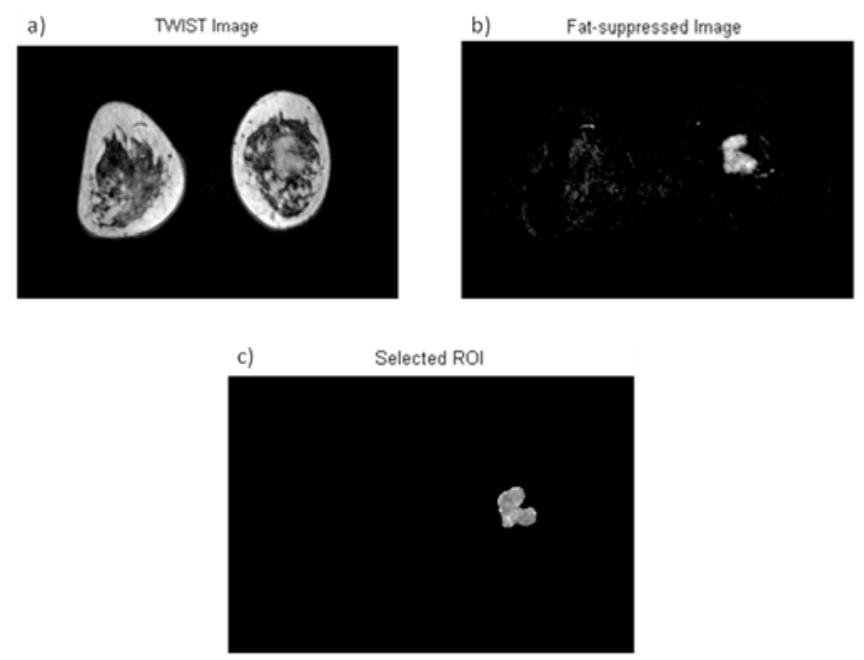

Figure 2. TWIST data: a) T1-w example image; b) Fat-suppressed image obtained subtracting the basal image from the 44th post contrast image; c) ROI selected by an expert radiologist. 
The selected ROIs included 1276 and 322 voxels for the TWIST and the FLASH data respectively.

Figure 3 shows the $C_{p}(t)$ fitting obtained using eq. 10 in TWIST data.

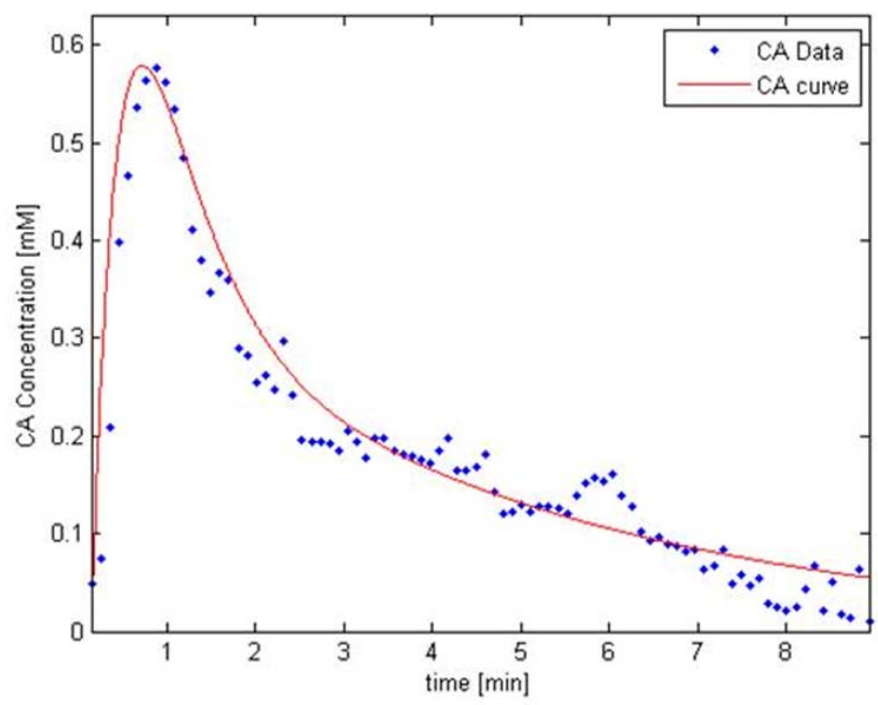

Figure 3. Manually selected AIF on TWIST data (blue dots) have been fitted by means of eq. 10 (red line).

Figure 4 reports some examples of the fitting obtained for Tofts, Brix and ATH for TWIST data (a) and the fitting for Tofts and Brix for FLASH data (b). For the curve in (a) the ATH model resulted in a better fitting in comparison to both Tofts and Brix (for both BIC and AIC); for the curve in (b) the Brix model showed a better fitting in comparison to the Tofts one.
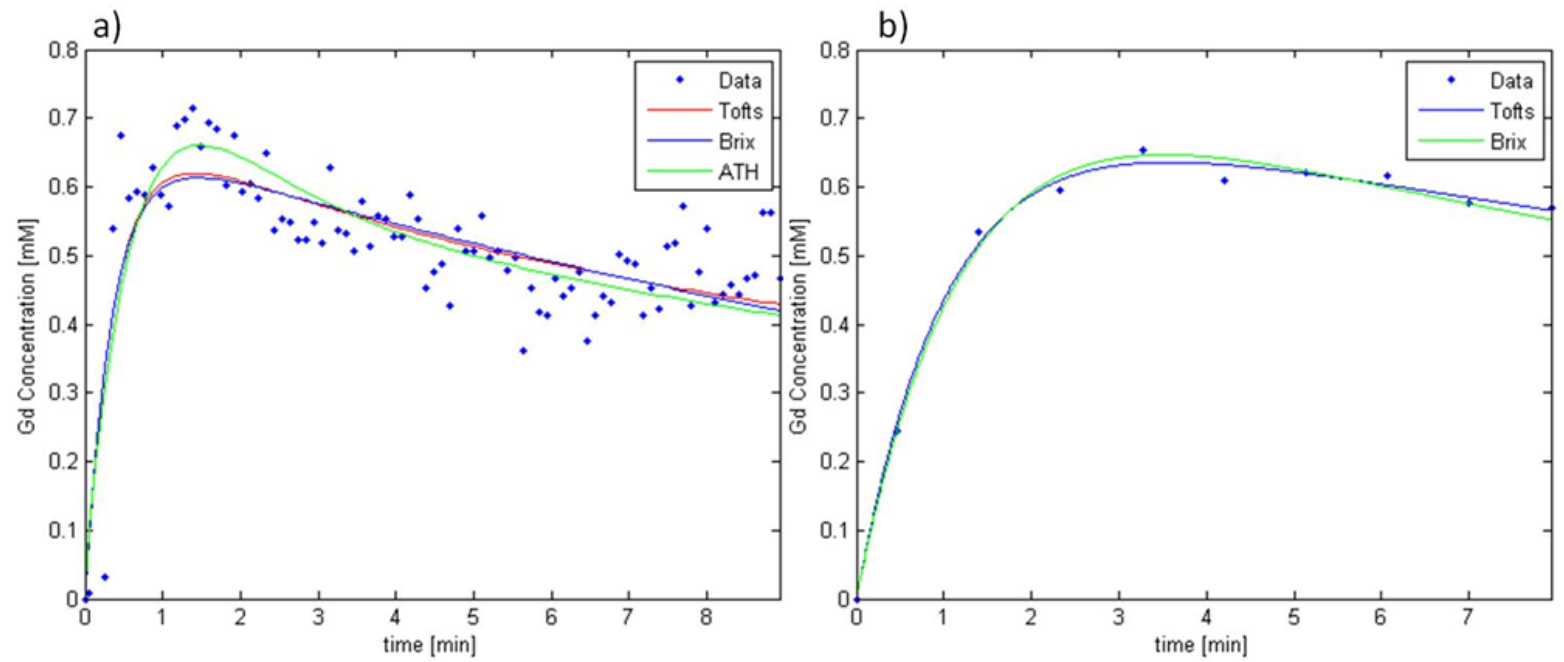

Figure 4. Example of data fitting. a) TWIST data (blue dots); Tofts model (red line); Brix model (blue line); ATH model (green line). b) FLASH data (blue dots); Tofts model (blue line); Brix model (green line).

The main results of this study are summarized in figures 5, 6 and in table 2 . 

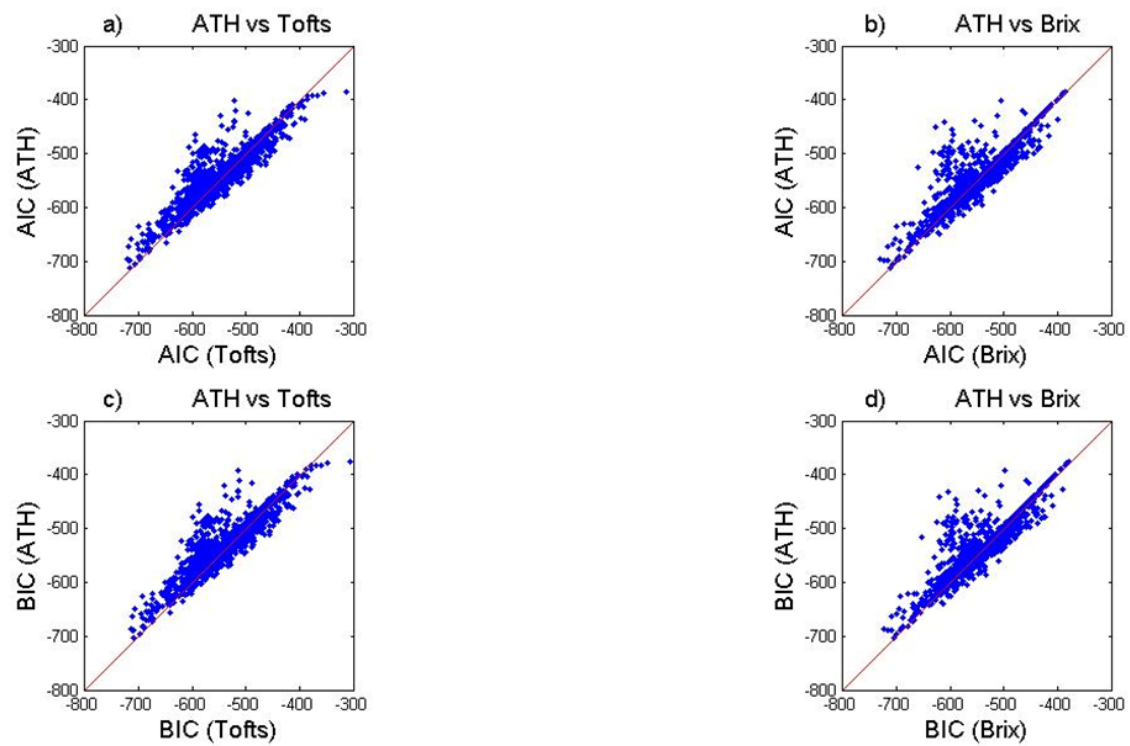

Figure 5. Goodness-of-fit for TWIST data. First row involves AIC: a) ATH versus Tofts; b) ATH versus Brix. Second row involves BIC: c) ATH versus Tofts; d) ATH versus Brix. Red lines indicate equal goodness of fit.

Table 2. Goodness-of-fit measurements on TWIST and FLASH data. Per each metric the table reports the percentage of voxels showing better fit of one model versus the other.

\begin{tabular}{llll}
\hline & ATH vs Tofts [\%] & ATH vs Brix [\%] & Tofts vs Brix [\%] \\
\hline$R_{1}^{2}<R_{2}^{2}($ TWIST) & - & - & 60 \\
$\mathrm{BIC}_{1}<\mathrm{BIC}_{2}$ (TWIST) & 65 & 64 & - \\
$\mathrm{AIC}_{1}<\mathrm{AIC}_{2}$ (TWIST) & 59 & 56 & - \\
$R_{1}^{2}<R_{2}^{2}$ (FLASH) & - & - & 77 \\
\hline
\end{tabular}

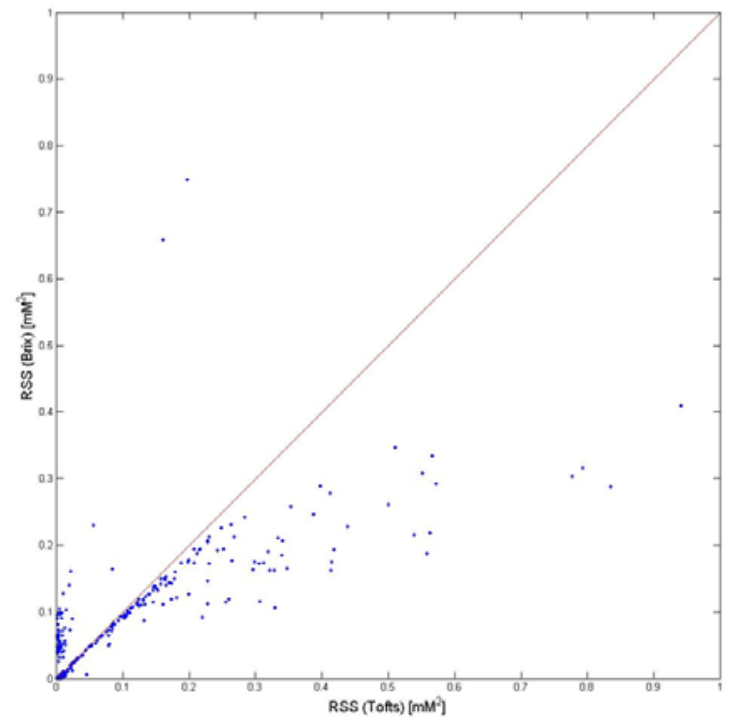

Figure 6. Goodness-of-fit for FLASH data. RSS values of Brix versus Tofts. 
Figure 5 reports the results of the goodness-of-fit analysis for TWIST data. With reference to the part (a) the graphs must be interpreted as follows. Each point represents a whole $\mathrm{C}_{\mathrm{t}}(\mathrm{t})$ : the value of the $\mathrm{x}$ - and $\mathrm{y}$-coordinates are the values of the AIC for Tofts and ATH model respectively. The red line indicate equal goodness-of-fit (identity). The points above the identity line denote cases in which the Tofts model gave better fit than ATH; points below lines denote cases in which ATH model gave better fit than Tofts. Similarly, figure 6 reports the results of RSS goodness-of-fit analysis for FLASH data. The interpretation of the figure is the same as figure 5 .

Table 2 reports, in the case of TWIST data, per each couple of models and per each metric (RSS, BIC, AIC), the percentage of voxels for which the metric computed for one model is lower than the same metric computed for the other model. Note that, as regards the comparisons among ATH, Tofts and Brix models we considered only the metrics BIC and AIC because the number of model parameters is different; on the contrary, in the case of Brix vs Tofts comparison we considered only the RSS. In the case of FLASH data, the comparison between Brix and Tofts models showed that $77 \%$ of voxels had lower RSS for Brix than for Tofts.

In order to evaluate consistency among the parameters estimated with the different models in table 3 and 4 we reported the median of each parameter on TWIST data and on FLASH data respectively. Moreover, with the aim to evaluate the reliability of the estimates, we reported also the median value of the standard deviations that have been calculated on the repetitions of Monte Carlo simulation.

Table 3. Reliability of fit parameters on TWIST data. Per each model the table reports the median value of each parameter \pm the median of standard deviation calculated on 100 ripetitions of the Monte Carlo simulation.

\begin{tabular}{|c|c|c|c|c|c|c|c|c|}
\hline & $\mathbf{K}^{\text {trans }}\left[\min ^{-1}\right]$ & $k_{e p}\left[\min ^{-1}\right]$ & $\mathbf{v}_{\mathbf{p}}$ & $\begin{array}{l}\text { A } \\
{[\mathrm{mmol} / \mathrm{kg}]}\end{array}$ & $k_{\mathrm{el}}\left[\min ^{-1}\right]$ & $\begin{array}{l}\mathrm{F}\left[\mathrm{mL} 100 \mathrm{~g}^{-1}\right. \\
\left.\mathrm{min}^{-1}\right]\end{array}$ & $\mathbf{E}$ & $\mathbf{T}_{\mathrm{c}}[\mathbf{s}]$ \\
\hline Tofts & $0.005 \pm 0.0001$ & $0.013 \pm 0.0001$ & $\begin{array}{l}0.097 \pm 0.02 \\
3\end{array}$ & & & & & \\
\hline Brix & & $0.031 \pm 0.003$ & & $\begin{array}{l}0.435 \pm 0.01 \\
5\end{array}$ & $\begin{array}{l}0.0002 \pm 0.000 \\
1\end{array}$ & & & \\
\hline ATH & $0.004 \pm 0.0001$ & $0.070 \pm 0.004$ & $\begin{array}{l}0.591 \pm 0.15 \\
5\end{array}$ & & & $0.099 \pm 0.033$ & $0.035 \pm 0.252$ & $6.001 \pm 0.00001$ \\
\hline
\end{tabular}

Table 4. Reliability of fit parameters on FLASH data. Per each model the table reports the median value of each parameter \pm the median of standard deviation calculated on 100 repetitions of the Monte Carlo simulation.

\begin{tabular}{llllll}
\hline & $\mathbf{K}^{\text {trans }}\left[\mathbf{m i n}^{-1}\right]$ & $\mathbf{k}_{\text {ep }}\left[\mathbf{m i n}^{-1}\right]$ & $\mathbf{v}_{\mathbf{p}}$ & $\mathbf{A}[\mathbf{m m o l} / \mathbf{k g}]$ & $\mathbf{k}_{\text {el }}\left[\mathbf{m i n}^{-1}\right]$ \\
\hline Tofts & $0.007 \pm 0.001$ & $0.010 \pm 0.0001$ & $0.002 \pm 0.027$ & & \\
Brix & & $0.016 \pm 0.004$ & & $0.678 \pm 0.074$ & $0.001 \pm 0.0004$ \\
\hline
\end{tabular}

\section{Discussion}

Many tracer kinetic models have been developed in the DCE-MRI context ${ }^{[11-25]}$. They can be roughly subdivided into $\mathrm{CC}^{[8-11,15]}$ and DP models ${ }^{[12,19,20]}$. Although DP models might be more realistic, to date most of clinical studies have been conducted using CC models ${ }^{[13,14,16,20-22]}$. In particular, in breast DCE-MRI commonly used were the Tofts ${ }^{[13,14,16]}$ and Brix models ${ }^{[20-22]}$; however, to the best of our knowledge, apart from a simulation study by Zwick et al. ${ }^{[13]}$, there is no study comparing them on real breast DCE-MRI data. Moreover, to date, there is no study explicitly comparing CC and DP models on real breast DCE-MRI data. Therefore, the objective of this study was two-fold: on the one hand we aimed to compare DP (ATH) to CC models (Tofts and Brix) on real breast DCE-MRI, on the other hand we compared Tofts to Brix on real breast DCE-MRI data. 
We analyzed breast DCE-MRI data from four subjects with histologically proven invasive ductal carcinoma; they underwent DCE-MRI examinations with two different sequences: TWIST and FLASH. The TWIST data were used for DP-CC comparison. This was done because the ATH model requires a short sampling interval lower than the mean capillary transit time $\left(\mathrm{T}_{\mathrm{c}}\right)$. FLASH data were used to perform the comparison between Brix and Tofts models in a typical clinical setting (as in previous studies). Models have been compared using different goodness-of-fit metrics (RSS, BIC, AIC).

We found that on TWIST data ATH obtained better fits than Tofts (59\% of voxels according to AIC and $65 \%$ according to BIC) and Brix (56\% of voxels according to AIC and 64\% according to BIC) although the percentages were not huge. The estimated parameters obtained with the different models were comparable and fell within a physiological range. As regards the reliability of the estimates it turned out that no model was capable of estimating all the parameters with reliability lower than about $10 \%$ and in some cases the reliability was very low.

In the analysis of FLASH data we found that Brix obtained better fit than Tofts in $77 \%$ of voxels. Also in this case the estimated parameters were comparable, however the reliability of the estimates obtained using Brix was higher than Tofts.

The better performances of ATH were in part expected considering that this model is in principle more realistic and that $\mathrm{C}_{\mathrm{p}}(\mathrm{t})$ has been derived from real data. However, the improvement in terms of goodness-of-fit was not decisive, probably because of several reasons: first, the temporal resolution achievable in current clinical settings could be not yet sufficient to satisfy the requirements on the capillary mean transit time; second, the typical signal-to-noise ratio achievable in current clinical setting could be not adequate to discriminate finer features of the CA time-course; finally, a major limitation of this study was the small number of subjects involved: this fact implies that the range of physiological parameters explored was limited, although they should be representative of important types of carcinomas, however it is worth to note that also in previous studies reporting comparison between models the number of subjects was small ${ }^{[28]}$.

As regards the comparison between Brix and Tofts it should be noted that, even if they have the same number of parameters, Brix includes a mono-exponential $C_{p}(t)$ within the model itself while Tofts uses a bi-exponential AIF. This could explain the better performance of Brix with respect to Tofts on FLASH data: at low temporal resolution the mono-exponential AIF is sufficient to capture essential features of the data. On the contrary, on TWIST data Tofts seems to behave better than Brix although the difference is not huge: at high temporal resolution the mono-exponential model becomes not adequate and the bi-exponential approach is required.

As regards the comparison between Tofts and Brix models our results are in line with recent literature. Zwick et al. ${ }^{[13]}$ reported the only comparison, to the best of our knowledge, on simulated DCE-MRI data and they concluded that Brix could be more robust than Tofts because it seems less affected by AIF variations.

As regards the comparison between DP and CC models conflicting results can be found in the literature. A theoretical study has been conducted by Muzic and Saidel ${ }^{[45]}$ on PET receptors: they concluded that CC models outputs yielded good fits to all the DP model outputs and the values of the corresponding parameters were in close agreement, but given the temporal resolution typically available with PET, the use of a DP model had no advantage over a CC model for PET receptor quantification. Another study by Cheong et al. ${ }^{[28]}$ reported a comparison of DP and CC models in DCE-Computerized Tomography (DCE-CT) of Intracranial Meningioma and concluded that DP models (including ATH) not only possess more realism theoretically but they were found to consistently give better fit than the CC models, although linear correlations were found between the kinetic parameters of the two models.

Our results do not allow to propend towards one model or the other: although DP models have been argued on physical and physiological grounds ${ }^{[12]}$, the question whether they can actually give better fit than CC models in breast DCE-MRI performed with current clinical settings, has not found yet a conclusive answer. 
One important issue in comparing models is the use of an appropriate criterion. In this study we adopted three commonly used indices having a common basis (Kullback-Leibler divergence ${ }^{[41]}$ ) and are largely accepted in the statistical community. We found that the results obtained with different indices were in agreement with each other.

\section{Conclusion}

To the best of our knowledge, this is the first evaluation of different kinetic models (conventional compartmental and distributed parameters) on breast DCE-MRI data. Although not conclusive, our results suggest that distributed parameters models (such as ATH) can achieve better fit than conventional compartmental ones (in particular Tofts and the Brix). Further investigation is required on this issue given the important clinical implications.

This study also provided a comparison, on the basis of real breast data, between Tofts and Brix model in clinical setting (low temporal resolution). The results indicate that the Brix model can achieve better fit in this case.

\section{References}

[1] Choyke PL, Dwyer AJ, Knopp MV. Functional Tumor Imaging With Dynamic Contrast-Enhanced Magnetic Resonance Imaging. J Magn Reson Imaging. 2003; 17: 509-52. PMid:12720260 http://dx.doi.org/10.1002/jmri.10304

[2] Collins J and Padhani R. Dynamic Magnetic Resonance Imaging of tumor Perfusion. IEEE Egineering in Medicine and Biology Magazine. 2004 September/October; 65-83.

[3] Padhani AR, Husband JE. Dynamic contrast- enhanced MRI studies in oncology with an emphasis on quantification, validation and human studies. Clin Radiol. 2001; 56: 607-620. PMid:11467863 http://dx.doi.org/10.1053/crad.2001.0762

[4] DeVries AF, Griebel J, Kremser C, et al. Tumor microcirculation evaluated by dynamic magnetic resonance imaging predicts therapy outcome for primary rectal carcinoma. Cancer Res. 2001; 61: 2513-2516. PMid:11289123

[5] Johansen R, Jensen LR, Rydland J, Goa PE, Kvistad KA, Bathen TF, Axelson DE, Lundgren S, Gribbestad IS. Predicting survival and early clinical response to primary chemotherapy for patients with locally advanced breast cancer using DCE-MRI. J Magn Reson Imaging. 2009 Jun; 29(6): 1300-7. PMid:19472387 http://dx.doi.org/10.1002/jmri.21778

[6] Ah-See ML, Makris A, Taylor NJ, Harrison M, Richman PI, Burcombe RJ, et al. Early changes in functional dynamic magnetic resonance imaging predict for pathologic response to neoadjuvant chemotherapy in primary breast cancer. Clin Cancer Res. 2008 Oct 15; 14(20): 6580-9. PMid:18927299 http://dx.doi.org/10.1158/1078-0432.CCR-07-4310

[7] Hoffmann U, Brix G, Knopp MV et al. Pharmacokinetic mapping of the breast: a new method for dynamic MR mammography. Magn Reson Med. 1994; 33: 506-514. PMid:7776881 http://dx.doi.org/10.1002/mrm.1910330408

[8] Brix G, Griebel J, Kiessling F, Wenz F. Eur J. Tracer kinetic modelling of tumour angiogenesis based on dynamic contrast-enhanced CT and MRI measurements. Nucl Med Mol Imaging. 2010 Aug; 37 Suppl 1: S30-51. PMid:20503049 http://dx.doi.org/10.1007/s00259-010-1448-7

[9] Bradley DP, Tessier JJ, Lacey T et al. Examining the acute effects of cediranib (RECENTIN, AZD2171) treatment in tumor models: a dynamic contrast-enhanced MRI study using gadopentate. Magn Reson Imaging. 2009; 27: 377-384. PMid:18814988 http://dx.doi.org/10.1016/j.mri.2008.07.021

[10] Chou CP, Wu MT, Chang HAT et al. Monitoring breast cancerresponse to neoadjuvant systemic chemotherapy using parametric contrast-enhanced MRI: a pilot study. Acad Radiol. 2007; 14: 561-573. PMid:17434070 http://dx.doi.org/10.1016/j.acra.2007.02.005

[11] Tofts PS. Modeling tracer kinetics in dynamic Gd-DTPA MR imaging. J Magnet Res Imaging. 1997; 7: 91-101. PMid:9039598 http://dx.doi.org/10.1002/jmri.1880070113

[12] St. Lawrence KS, Lee T-Y. An adiabatic approximation to the tissue homogeneity model for water exchange in the brain: I. theoretical derivation. J Cerebral Blood Flow Metab. 1998; 18: 1365-1377. http://dx.doi.org/10.1097/00004647-199812000-00011

[13] Zwick S, Brix G, Tofts PS, Strecker R, Kopp-Schneider A, Laue H, Semmler W, Kiessling F. Simulation-based comparison of two approaches frequently used for dynamic contrast-enhanced MRI. Eur Radiol. 2010 Feb; 20(2): 432-42. PMid:19727758 http://dx.doi.org/10.1007/s00330-009-1556-6

[14] Sansone M, Fusco R, Petrillo M, Petrillo A, Bracale M. An expectation maximisation approach for simultaneous pixel classification and tracer kinetic modelling in dynamic contrast enhanced-magnetic resonance imaging. Med Biol Eng Comput. 2011 Apr; 49(4): 485-95. http://dx.doi.org/10.1007/s11517-010-0695-x 
[15] Brix G, Semmler W, Port R. Phrmacokinetic Parameters in CNS Gd-DTPA enhanced MR imaging. J. Comput Assist Tomogr. 1991; 15: 621-628. PMid:2061479 http://dx.doi.org/10.1097/00004728-199107000-00018

[16] Ahearn TS, Sta RT, Redpath TW, Semple SIK. The use of the LevenbergMarquardt curve fitting algorithm in pharmacokinetic modelling of DCE-MRI data. Phys Med Biol. 2005; 50: N85-N92.

[17] Johnson JA, Wilson TA. A model for capillary exchange. Am J Physiol. 1966; 210: 1299-1303. PMid:5923068

[18] Larson KB, Markham J, Raichle ME. Tracer kinetic models for measuring cerebral blood flow using externally detected radiotracers. J Cereb Blood Flow Metab. 1987; 7: 443-463. http://dx.doi.org/10.1038/jcbfm.1987.88

[19] Koh TS, Cheong LH, Hou Z, Soh YC. A physiologic model of capillary-tissue exchange for dynamic contrast-enhanced imaging of tumor microcirculation. IEEE Trans Biomed Eng. 2003; 50: 159-167. PMid:12665029 ttp://dx.doi.org/10.1109/TBME.2002.807657

[20] Aaron RK, Dyke JP, Ciombor DM et al. Perfusion abnormalities in subchondral bone associated with marrow edema, osteoarthritis, and avascular necrosis. Ann N Y Acad Sci. 2007; 1117: 124-137. PMid:18056039 http://dx.doi.org/10.1196/annals.1402.069

[21] Chun H, Clymer B, Sammet S et al. Improvement in the reproducibility of region of interest using an auditory feedback loop: a pilot assessment using dynamic contrast-enhanced (DCE) breast MR images. J Magn Reson Imaging. 2008 ; 27 : 27-33. PMid:18058928 http://dx.doi.org/10.1002/jmri.21229

[22] Giesel FL, Choyke PL, Mehndiratta A et al. Pharmacokinetic analysis of malignant pleural mesotheliomainitial results of tumor microcirculation and its correlation to microvessel density (CD-34). Acad Radiol. 2008; 15: 563-570. PMid:18423312 http://dx.doi.org/10.1016/j.acra.2007.12.014

[23] Huijun Chen, Feiyu Li, Xihai Zhao, Chun Yuan,Brian Rutt and William S. Kerwin1. Extended Graphical Model for Analysis of Dynamic Contrast-Enhanced MRI. Magnetic Resonance in Medicine. 2011; 66: 868-878. PMid:21394770 http://dx.doi.org/10.1002/mrm.22819

[24] Donaldson SB, West CML, Davidson SE, Carrington BM, et al. A Comparison of Tracer Kinetic Models for T1-Weighted Dynamic Contrast-Enhanced MRI: Application in Carcinoma of the Cervix. Magnetic Resonance in Medicine. 2010; 63: 691-700. PMid:20187179 http://dx.doi.org/10.1002/mrm.22217

[25] Weinmann HJ, Laniado M, Mutzel W. Pharmacokinetics of GdDTPA/dimeglumine after intravenous injection into healthy volunteers. Physiol Chem Phys Med NMR. 1984; 16: 167-12. PMid:6505043

[26] Schabel MC, Morrell GR, Oh KY, Walczak CA, Barlow RB, Neumayer LA. Pharmacokinetic mapping for lesion classification in dynamic breast MRI. J Magn Reson Imaging. 2010 Jun; 31(6): 1371-8. PMid:20512889 http://dx.doi.org/10.1002/jmri.22179

[27] Orton MR, d'Arcy JA, Walker-Samuel S, Hawkes DJ, Atkinson D, Collins DJ, et al. Computationally efficient vascular input function models for quantitative kinetic modelling using dee-mri, Physics in Medicine and Biology. 2008; $53(5)$ : 1225. PMid:18296759 http://dx.doi.org/10.1088/0031-9155/53/5/005

[28] Dennis Cheong LH, Tchoyoson Lim CC, Koh TS. Dynamic contrast-enhanced CT of intracranial meningioma: comparison of distributed and compartmental tracer kinetic models - initial results. Radiology. 2004 Sep; 232(3): 921-30. PMid:15247436 http://dx.doi.org/10.1148/radiol.2323031198

[29] Li X, Welch EB, Chakravarthy AB, Xu L, Arlinghaus LR, Farley J, et al. Statistical comparison of dynamic contrast-enhanced MRI pharmacokinetic models in human breast cancer. Magn Reson Med. 2011 Nov 29.

[30] Song T et al., Optimal k-space sampling for dynamic contrastenhanced MRI with an application to MR renography, Magn Reson Med. 2009; 61(5): 1242-1248. PMid:19230014 http://dx.doi.org/10.1002/mrm.21901

[31] Haase A, Frahm J, Matthaei D, Hanicke W, Merboldt KD. FLASH imaging - rapid NMR imaging using low flip-angle pulses. J Magn Reson. 1986; 67: 258-266.

[32] Stanisz GJ, Henkelman RM. Gd-DTPA relaxivity depends on macromolecular content. Magn Reson Med. 2000 Nov; $44(5)$ : 665-7. http://dx.doi.org/10.1002/1522-2594(200011)44:5<665::AID-MRM1>3.0.CO;2-M

[33] Fram EK, Herfkens RJ, Johnson GA, Glover GH, Karis JP, Shimakawa A, et al. Rapid calculation of T1 using variable flip angle gradient refocused imaging. Magn Reson Imaging. 1987; 5: 201-208. http://dx.doi.org/10.1016/0730-725X(87)90021-X

[34] Kety SS. The theory and applications of the exchange of inert gas at the lungs and tissues. Pharmacol Rev. 1951; 3: 1-41. PMid:14833874

[35] Yankeelov TE, Cron GO, Addison CL, Wallace JC, Wilkins RC, Pappas BA, et al. Comparison of a reference region model with direct measurement of an AIF in the analysis of DCE-MRI data. Magn Reson Med. 2007 Feb; 57(2): 353-61. PMid:17260371 http://dx.doi.org/10.1002/mrm.21131

[36] Walker-Samuel S, Leach MO, Collins DJ. Reference tissue quantification of DCE-MRI data without a contrast agent calibration. Phys Med Biol. 2007 Feb 7; 52(3): 589-601. PMid:17228107 http://dx.doi.org/10.1088/0031-9155/52/3/004 
[37] Lehman CD, Blume JD, Weatherall P, Thickman D, Hylton N, Warner E, et al. Screening women at high risk for breast cancer with mammography and magnetic resonance imaging. Cancer. 2005; 103 (9):1 898-1905.

[38] Cheng M. Investigation and Optimization of Parameters Accuracy in Dynamic Contrast-Enhanced MRI. Journal of Resonance Imaging. 2008; 28: 736-743. PMid:18777534 http://dx.doi.org/10.1002/jmri.21489

[39] Koh TS, Zeman V, Darko J, Lee TY, Milosevic MF, Haider M, et al. The inclusion of capillary distribution in the adiabatic tissue homogeneity model of blood flow. Phys Med Biol. 2001 May; 46(5): 1519-38. PMid:11384068 http://dx.doi.org/10.1088/0031-9155/46/5/313

[40] Colin Cameron, A.; Windmeijer, Frank A.G.; Gramajo, H; Cane, DE; Khosla, C. An R-squared measure of goodness of fit for some common nonlinear regression models. Journal of Econometrics. 1997; 77 (2): 1790-2. http://dx.doi.org/10.1016/S0304-4076(96)01818-0

[41] Akaike H. A new look at the statistical model identification. IEEE Trans Auto Control. 1974; 19: 716-723. http://dx.doi.org/10.1109/TAC.1974.1100705

[42] Bevington PR. Data reduction and error analysis for the physical sciences. New York, NY: McGraw-Hill, 1969.

[43] Simpson, NE. \& He, Z \& Evelhoch JL. Deuterium NMR tissue perfusion measurements using the tracer uptake approach: I. Optimization of methods. Magn Reson Med. 1999; 42: 42-52. http://dx.doi.org/10.1002/(SICI)1522-2594(199907)42:1<42::AID-MRM8>3.0.CO;2-Z

[44] Li J, Yu Y, Zhang Y, Bao S, Wu C, Wang X, et al. A clinically feasible method to estimate pharmacokinetic parameters in breast cancer. Med Phys. 2009 Aug; 36(8): 3786-94. PMid:19746812 http://dx.doi.org/10.1118/1.3152113

[45] Muzic RF Jr, Saidel GM. Distributed versus compartment models for PET receptor studies. IEEE Trans Med Imaging. 2003; 22: 11-21. PMid:12703756 http://dx.doi.org/10.1109/TMI.2002.806576

[46] Antoine Rosset, Luca Spadola and Osman Ratib OsiriX: An Open-Source Software for Navigating in Multidimensional DICOM Images. J Digit Imaging. 2004; 17(3): 205-216. PMid:15534753 http://dx.doi.org/10.1007/s10278-004-1014-6 